Cahiers d'études africaines

$234 \mid 2019$

Le politique, une affaire de famille?

\title{
Les conflits de proximité et la crise de la démocratie au Niger : de la famille à la classe politique
}

Rivalries of Proximity and the Crisis of Democracy: From Household to Political

Elites, in Niger and Beyond

Jean-Pierre Olivier de Sardan

\section{OpenEdition}

Journals

Édition électronique

URL : https://journals.openedition.org/etudesafricaines/25853

DOI : 10.4000/etudesafricaines.25853

ISSN : $1777-5353$

Éditeur

Éditions de l'EHESS

Édition imprimée

Date de publication : 15 juin 2019

Pagination : 405-425

ISSN : 0008-0055

Référence électronique

Jean-Pierre Olivier de Sardan, « Les conflits de proximité et la crise de la démocratie au Niger : de la famille à la classe politique », Cahiers d'études africaines [En ligne], 234 | 2019, mis en ligne le 01 janvier 2022, consulté le 21 avril 2022. URL : http://journals.openedition.org/etudesafricaines/25853 ; DOI : https://doi.org/10.4000/etudesafricaines.25853 


\section{Les conflits de proximité et la crise de la démocratie au Niger: de la famille à la classe politique}

Si la politique est, en Afrique (mais ailleurs également), bien souvent une affaire de famille, la famille y est de son côté, bien souvent, une affaire de politique, en ce sens que la gestion des enjeux de pouvoir et des jalousies conjugales ou fraternelles est au cœur des relations familiales. Autrement dit, famille et politique ont certaines propriétés structurelles communes, qui se situent, selon nous, dans le registre des conflits de proximité, et que nous souhaitons ici explorer ${ }^{1}$.

Cette perspective va à l'encontre de clichés largement répandus qui opposent la famille comme espace de solidarité interne à la politique comme espace d'affrontements externes. Cette perspective est doublement contestable : les relations familiales sont certes publiquement de l'ordre de l'entraide et de la fraternité, mais la réalité quotidienne est bien différente. Inversement, les compétitions frontales entre camps politiques masquent le fait que chaque camp est lui aussi traversé de rivalités et de tensions, mais de rivalités internes et de tensions feutrées, entre camarades, entre amis, entre alliés. Comme la famille!

Néanmoins, l'analyse des conflits politiques reste largement focalisée sur les conflits entre adversaires qu'une "frontière » sépare, que cette frontière soit religieuse, sociale, ethnique, culturelle, nationale, idéologique... Ce sont alors des conflits de distance. Les exemples dans la littérature académique sont innombrables. L'hostilité entre deux camps est fondée sur le choc de deux identités respectives, qui se présentent comme irréductibles l'une à l'autre, ou sur l'affrontement de deux logiques antagonistes. Les conflits de proximité constituent un tout autre type de conflits, qui oppose des adversaires

1. Je remercie Eric Hahounou, Abdoulaye Sounaye et Mahaman Tidjani Alou pour leurs commentaires. Une première version des arguments ici développés a été publiée dans OLIVIER DE SARDAN (2017). Elle a été significativement remaniée et considérablement développée dans le présent texte. 
caractérisés au contraire par leur similitude. L'antagonisme intervient entre parents, entre proches.

Dans les conflits de distance, l'un combat l'autre parce qu'il est socialement construit comme un Autre. Ces conflits sont ouverts, publics. Ils opposent des dissemblables. Dans les conflits de proximité, l'un est rival de l'autre parce qu'il est socialement construit comme le Même. Ces conflits sont discrets, privés. Ils opposent des semblables.

La proximité peut être familiale comme elle peut être politique. Ce sont ces conflits de proximité que nous analyserons ici, en nous appuyant sur le cas du Niger, où ils prennent une particulière importance.

Le contexte politique nigérien

En effet, la vie politique du Niger est dominée, depuis la Conférence nationale en 1991, qui a introduit le multipartisme après trente années de parti unique puis de dictature militaire, par deux phénomènes proches : (a) d'une part le «nomadisme politique» (les militants et les cadres circulent sans cesse d'un parti à l'autre, au rythme soutenu des factions et des scissions); (b) d'autre part la « valse des alliances » (tous les partis se sont mutuellement alliés puis combattus, puis alliés, puis combattus, au fil des années). Ce sont les leaders politiques qui ont émergé lors de la Conférence nationale qui dirigent encore aujourd'hui tous les principaux partis, tous ayant alterné au pouvoir comme dans l'opposition dans toutes les configurations possibles.

Une même classe politique se succède à elle-même sans cesse depuis 25 ans, entre compromis et bras de fer, réconciliations et divorces, fraternisation et haine. Ils dénoncent tous, quand ils sont (à tour de rôle) dans l'opposition, les comportements qu'ils adoptent tous quand ils sont (à tour de rôle) au pouvoir (politisation de l'administration, interventionnisme permanent de type clientéliste dans le fonctionnement des services publics, enrichissement accéléré des dignitaires du régime et de leurs protégés, manipulation des élections, persécution de l'opposition...) $)^{2}$.

Cette classe politique se compose en fait de quelques milliers de personnes, qui se connaissent tous personnellement. Une partie importante (et la plus visible) de cette classe politique est composée d'élites instruites, qui ont souvent fréquenté les mêmes écoles et qui appartiennent dans leur majorité aux " grandes familles » du Niger. Cette notion de " grande famille» est émique, elle traduit exactement l'expression windi beeri, utilisée couramment en langue songhay-zarma pour désigner les familles importantes, éminentes,

2. Voir notre article sur « Les prisons du pouvoir» (OLIVIER DE SARDAN 2016). 
respectables, autrement dit relevant de l'aristocratie ou de la bourgeoisie, par opposition aux "petites familles» (windi kaina), celles du peuple, des pauvres et des dominés (talaka) ${ }^{3}$. Cette classe politique restreinte a toutes les caractéristiques d'une bourgeoisie politico-économique d'interconnaissance, unie par un même mode de vie, la fréquentation des mêmes lieux, et de multiples alliances matrimoniales : accumulation grâce à la corruption et à l'affairisme, investissements dans l'immobilier et l'élevage, dépenses somptuaires et redistributions clientélistes, multiplication des privilèges de fonction, absence de base idéologique pour les clivages partisans, placement des enfants dans les mêmes écoles privées élitistes et des universités du Nord, soins et évacuations sanitaires à l'étranger, intermariages quasi systématiques, fréquentation mutuelle et sociabilité partagée au fil des baptêmes et noces qui rythment la vie sociale de la capitale, etc. Néanmoins, au sein de cet entrelac de relations mutuelles et croisées, les batailles sont féroces entre les ennemis d'aujourd'hui, qui sont les alliés d'hier, et peut-être ceux de demain. Tous les coups, ou presque, sont permis, l'emprisonnement d'un ancien camarade étant la forme la plus exacerbée des règlements de comptes ${ }^{4}$.

Cette classe politique est indissociable de la démocratie dont elle tient tous les leviers. La démocratie avait suscité à ses débuts beaucoup d'espoir. Mais désormais c'est, de loin, la déception qui domine, dans tous les secteurs de la société, face aux jeux politiciens incessants, aux alliances se modifiant à 180 degrés du jour au lendemain, aux rivalités personnelles exacerbées et aux épreuves de force incessantes, aux luttes de cliques et de clans, le tout sur un fond de faible délivrance des services publics, d'enrichissement spectaculaire des élites, de chômage massif des jeunes, et de corruption généralisée. On assiste donc à un rejet de plus en plus répandu de la «politik» (ce terme est désormais passé en langue songhay-zarma, avec un contenu très péjoratif),

3. Ces grandes familles sont au carrefour de la chefferie, de l'enrichissement économique et du capital scolaire. Voir Tidjani Alou (1992) pour une description du rôle joué par l'école coloniale dans la production de cette élite restreinte. Toutefois, il faut noter que la classe politique inclut désormais des «parvenus » ou des grands commerçants roturiers, parfois analphabètes, qui financent les partis, les campagnes électorales, et les leaders politiques. Ils ont non seulement des relations «d'affaires » avec les élites instruites, mais ils partagent aussi avec elles une forte sociabilité (mariages, baptêmes, funérailles, cérémonies islamiques, réceptions protocolaires).

4. On se rappellera que, lors de la dernière élection présidentielle, Hama Amadou, qualifié pour le second tour et principal opposant du président Mamadou Issoufou, était emprisonné pendant la campagne électorale. Pourtant, plusieurs fois au cours des trente dernières années, Hama Amadou et Mamadou Issoufou ont été alliés. Même si ce cas est extrême, le passage par la case « prison» se retrouve dans les parcours d'un nombre non négligeable de personnalités politiques nigériennes (pour des motifs invoqués divers, mais en particulier préparation de coup d'État ou détournement de fonds). 
sous-entendu la politique des partis, la politique des politiciens, la politique de la démocratie. La démocratie est vécue désormais comme un échec. La nostalgie croissante envers le régime militaire de Seyni Kountché (idéalisé a posteriori et crédité de rigueur morale et civique et de sens du service public en opposition aux mœurs politiques actuels) en est un des signes.

Une autre conséquence, bien plus dramatique, de ce rejet de la politique démocratique telle qu'elle se manifeste au Niger (et de la classe politique qui l'a mise en œuvre), est la progression spectaculaire de l'idéologie salafiste, mais aussi, en son sein, des opinions extrémistes islamistes. Les réseaux ou groupes islamistes fondamentalistes, par leur critique impitoyable du système en place et leur proposition d'une alternative radicale fondée sur la charia, mais aussi parfois sur le caliphat et même, à l'extrême, sur le jihad (cf. Boko Haram dans le Sud-Est nigérien, ou le Mujao dans le Nord-Ouest), profitent de cette crise de la démocratie (qui est aussi à divers égards une crise de la société nigérienne elle-même) au même titre que le font les groupes d'extrême droite en Europe. L'islamisme radical est en quelque sorte le vecteur idéologique d'un « dégagisme » nigérien, qui veut en finir avec le régime des partis et le pouvoir des politiciens.

Au-delà de ces positions extrêmes, la méfiance envers la politik est désormais profonde au sein de la population nigérienne, et l'une des principales raisons invoquées par tous est la récurrence des rivalités internes à la classe politique, la domination des comportements de baab-izey (voir infra). Notre propos ici portera sur la façon dont ces rivalités s'expriment dans le langage quotidien, et les configurations sémantiques locales qui en rendent compte. En-fait, il y a un lien direct entre les perceptions sociales des rivalités intrafamiliales et les perceptions sociales des rivalités politiques. Un même ensemble sémiologique est mobilisé pour penser les rivalités de proximité, que celles-ci relèvent de l'univers de la parenté, ou qu'elles interviennent dans l'univers du pouvoir. En outre, au-delà de ces logiques représentationnelles similaires, certaines logiques symboliques et pragmatiques analogues traversent aussi ces deux univers, avec un recours intensif à des entrepreneurs magico-religieux qui tirent profit de ces rivalités et, de surcroît, les alimentent.

Notre argumentation s'appuie essentiellement sur le Niger, et sur une expérience de cinquante années de recherches dans ce pays, dont plus d'une vingtaine d'années vécues sur place. Elle utilise des exemples pris dans la langue songhay-zarma, que nous parlons. Mais les autres langues du Niger connaissent des expressions et des catégorisations similaires ${ }^{5}$. Et, plus largement, les pays sahéliens voisins ne sont pas sans témoigner de rivalités de proximité analogues. Ils ont en effet de nombreux points communs avec

5. Par exemple dan uwa/dan uba en hausa, bi baba/bi ina en peul. 
le Niger, au niveau politique (une classe politique similaire, une même perte de crédibilité de la démocratie), religieux (importance de l'islam, montée du salafisme, et rôle central souterrain des entrepreneurs magico-religieux), culturel (parenté à plaisanterie, polygamie), linguistique (existence de langues transversales aux frontières nationales comme le peul, le bambara, le soninké, le tamashek, le songhay) ou social (fossé entre peuple et élites). Sous réserve de vérifications empiriques, il semble que l'analyse développée ici pourrait être étendue assez facilement aux contextes proches, comme le Mali, le Burkina Faso ou le Sénégal.

\section{Un peu de sémiologie populaire songhay-zarma}

Au Niger, en langue songhay-zarma, deux termes sont couramment utilisés pour décrire de façon très dépréciative les dimensions conflictuelles de la politique (politik). L'un se rattache à une tradition musulmane, l'autre à une tradition locale. Il s'agit d'une part de fitina, la discorde (terme d'origine arabe); et d'autre part de baab-ize tarey, la jalousie et la compétition (littéralement : la condition d'enfants de même père et de mères différentes) ${ }^{6}$.

Fitina connote toutes les rivalités de proximité, à partir d'un univers sémantique islamique. Le mot s'applique en effet originellement aux divisions entre musulmans, autrement dit au fait (diabolique) d'introduire la discorde au sein de la communauté des croyants (umma). Dans le langage courant, il s'est étendu à la communauté politique (qui, au Niger, est aussi pour l'essentiel faite de musulmans) et aux conflits qui la caractérisent.

Mais c'est la seconde expression, issue de l'univers sémantique de la parenté, et qui revient de façon récurrente dans le langage quotidien, qui nous intéressera ici et que nous allons expliciter.

Les baab-izey (baaba, père ; izey, enfants ; autrement dit, littéralement, les fils ou filles de père) s'opposent aux nya-izey (nya, mère; autrement dit, littéralement, les fils ou filles de mère), dans un contexte essentiellement polygame. Les nya-izey (parfois aussi appelés fu-follon-izey, enfants d'une seule maisonnée) sont les enfants d'un même père et d'une même mère, soudés autour de cette dernière, alors que les baab-izey sont les enfants d'un même père mais de mères différentes. La configuration familiale la plus fréquente se compose en effet, sous l'autorité du père, de plusieurs foyers maternels :

6. Pour un aperçu en profondeur des concepts et conceptions songhay-zarma relatifs au pouvoir ou à la famille, voir OLIVIER DE SARDAN (1982). Pour une analyse historique de la transformation sous la colonisation des rapports sociaux pré-coloniaux, voir OLIVIER DE SARDAN (1984). 
les frères et sœurs au sein d'un de ces foyers («même père-même mère » dit-on dans le parler français du Niger) sont entre eux des nya-izey, alors que les demi-frères et demi-sœurs de différents foyers maternels mais de même père sont baab-izey les uns par rapport aux autres?

Dans les représentations populaires, le rapport archétypique des nya-izey entre eux est un rapport de complicité et d'entraide, alors que le rapport archétypique des baab-izey entre eux est un rapport de compétition et de jalousie ${ }^{8}$. Les solidarités intra-familiales en Afrique ont été depuis longtemps soulignées dans la littérature savante, elles font partie de l'idéologie dominante, elles sont affichées, et elles ont bien sûr une existence réelle. Mais les antagonismes intra-familiaux n'en existent pas moins eux aussi, concomitamment, même s'ils sont moins mentionnés et bien peu analysés.

\section{De la sémiologie aux pratiques domestiques}

Cette opposition baab-izey/nya-izey repose sur trois piliers sociaux ancrés dans les réalités quotidiennes.

Le premier est la rivalité latente qui oppose les co-épouses entre elles, pour gagner les faveurs ou les présents du mari, et s'affirmer chacune aux dépens des autres ${ }^{9}$. On pourrait même parler d'un «syndrome de la co-épouse», qui fait qu'une femme voit dans toute autre femme une concurrente ou une menace, et qui s'étend même au-delà des contextes polygames stricto sensu (dans un couple monogame, le mari est presque toujours soupçonné de vouloir prendre une autre épouse ; un mari qui a divorcé et s'est remarié est bien souvent suspecté d'entretenir des relations ambigües avec son ancienne femme,

7. L'ensemble des enfants d'un même père est appelé baaba-fo-izey (« enfants d'un père »). Si ce père a eu des enfants de plusieurs femmes, cet ensemble pourra donc être composé de plusieurs sous-ensembles de nya-izey, qui sont liés les uns aux autres par des relations de baab-izey.

8. Les langues voisines connaissent la même opposition : par exemple $f a-d e / b a-d e$ en bambara. Dans la réalité quotidienne, cette opposition, qui est un modèle émique, n'empêche évidemment pas l'existence éventuelle de rapports conflictuels entre nya-izey et de rapports affectueux entre baab-izey.

9. Les exemples, en particulier dans les campagnes, de cas (souvent cités par les tenants de la polygamie) où une épouse souhaite avoir une co-épouse pour partager les tâches ménagères, ou encore où deux co-épouses s'entendent bien, ne mettent pas en cause la validité générale du modèle émique de la rivalité entre co-épouses (et plus encore en ville et au sein des élites et classes moyennes). 
et les enfants d'un premier lit sont vite perçus comme des menaces par la nouvelle épouse) ${ }^{10}$.

Le second renvoie à la succession et à l'héritage, et aux préférences dont un père peut témoigner, à travers ses dons, ses choix ou ses legs, à l'égard de tel ou tel de ses enfants, préférences qui seront vécues comme insupportables par ceux de ses enfants qui ont une autre mère que celle de l'élu(e).

Le dernier est spécifique à un milieu social très particulier, celui de la chefferie, mais il irrigue toute la société, tant les affaires de chefferie sont au centre des espaces publics ruraux, et même de l'espace public national. En effet, depuis les débuts de la colonisation (création de la chefferie administrative), les chefs de canton (ou les chefs de groupements en zone dite nomade) sont choisis (par le pouvoir) ou élus (par les chefs de village) au sein de la famille « régnante », autrement dit parmi les «ayant-droits » ayant fait acte de candidature. Ces « ayant-droits » sont les descendants (en filiation patrilinéaire) des premiers chefs de canton. Autrement dit, les candidats rivaux sont tous « frères » (ou cousins parallèles) entre eux (Tidjani Alou 2009) ${ }^{11}$. Ce choix, ou, depuis quelques décennies, cette élection, suscite une lutte souvent féroce entre les «baab-izey» de la famille du chef, qui sont en compétition sourde ou ouverte pour cette charge à vie. Le demi-frère ou le cousin battu deviendra souvent un opposant à vie de son parent devenu chef de canton (Olivier de Sardan 2009).

On voit que la polygamie est au cœur de ces différents processus. Les baab-izey sont des enfants de co-épouses, qui reproduisent les rivalités de leurs mères. La polygamie alimente un conglomérat de jalousies et de conflits qui prennent place dans l'espace domestique (compétition entre co-épouses et entre leurs enfants respectifs), et parfois au sein de l'espace politique de la chefferie (dans la mesure où celui-ci est co-extensif à l'espace domestique de la famille au pouvoir).

10. Voir la description des rivalités entre co-épouses dans FAINZANG \& JoURnET (1988), à propos des Toucouleurs et des Soninkés au Sénégal.

11. Les cousins parallèles (enfants respectifs de deux frères ou de deux sœurs) sont appelés « frères » ou « sœurs » en songhay-zarma : le vocabulaire commun ne fait pas de différence entre eux et les frères directs; de même, les oncles paternels ou les tantes maternelles des cousins parallèles sont appelés par ceux-ci «père » (baaba) ou «mère» (nya). En revanche, les cousins croisés (enfants respectifs d'un frère et d'une sœur) sont appelés baasey, la tante paternelle haawey et l'oncle maternel haasey; les relations entre cousins croisés sont le noyau des relations de plaisanterie (appelées "baaso-tarey ", "condition de cousins croisés »), qui sont en vigueur au-delà de la famille. Enfin, il y a une nette différence au sein d'une fratrie (y inclus cousins parallèles) entre les ainés - beerey, grands frères ou grandes sœurs - et les cadets - kayney, petits frères ou petites sœurs - (alors que, en ce qui concerne les cousins croisés, ce sont les enfants du frère qui ont préséance sur les enfants de sa sœur). 
Des stéréotypes sur la famille africaine aux normes pratiques familiales

Ces représentations et ces pratiques sont en contradiction évidente avec nombre de stéréotypes sur « la famille africaine », qui serait avant tout un lieu d'entraide et de solidarités. Ces clichés sont souvent le fait d'une idéologie plus ou moins «primitiviste» et « communautariste», d'origine occidentale (Courade 2006), mais ils correspondent également pour une part à des valeurs proclamées par la morale familiale et sociale locale. En effet, selon les normes sociales qui régulent officiellement la famille polygame, toutes les co-épouses sont égales, et chacune traite les enfants des autres comme les siens propres. Cette attitude de non-discrimination qu'une mère doit adopter entre ses propres enfants et les enfants de ses co-épouses, lesquels de leur côté doivent la considérer eux-mêmes comme une mère à l'égal de leur mère biologique, est très souvent mise en avant dans les discours publics et les propos tenus aux étrangers, avec le recours à diverses anecdotes pour attester de ce que cette norme sociale est bel et bien intériorisée et appliquée (du type : «jusqu'à l'âge de 10 ans je n'ai pas su qui était ma vraie mère»...). Cette norme sociale prend d'ailleurs parfois aussi une tonalité religieuse, en référence aux passages du Coran préconisant un traitement égalitaire au sein de la famille polygame ${ }^{12}$.

Mais qui peut croire que les normes sociales sont toujours appliquées, et que leur observance est un fait courant, normal et banal? Les normes familiales, comme les normes religieuses ou les normes de voisinage, sont certes proclamées, relèvent de valeurs morales affirmées et font l'objet d'une éducation publique. Mais elles sont aussi, dans la pratique, souvent contournées, au profit de dérives, de ruses et d'arrangements informels. Toutefois, ces écarts aux normes sociales ne sont pas pour autant anarchiques, et restent régulés de facto, en sourdine. Autrement dit, sous une norme sociale explicite, on trouve le plus souvent des «normes pratiques » implicites qui, de façon plus ou moins tacite et dissimulée, organisent ou routinisent les comportements fréquents qui s'éloignent de la norme sociale affichée, autrement dit

12. Mais l'islam n'aborde le problème de l'égalité des co-épouses que du point de vue de l'homme : il en reconnait la difficulté, puisque ne devrait être polygame que celui qui peut, à l'image du Prophète, traiter chaque épouse de la même façon: c'est la fameuse sourate An Nisa (Coran 4:3) : «Des femmes qui te semblent bonnes, épouses-en deux ou trois ou quatre; et si tu crains de ne pas les traiter de façon équitable, tu t'en tiendras à une seule. » Cette norme religieuse qui, très exigeante en son principe, est évidemment bien loin d'être respectée dans la pratique quotidienne des croyants... 
qui régulent les comportements «non observants ${ }^{13}$. Bien évidemment, une femme ne va pas brimer les enfants de ses co-épouses ou leur parler mal devant le père ou un visiteur. En situation publique, la norme sociale règne, elle est ostentatoirement respectée. C'est dans l'intimité, loin des regards, que la norme pratique sera mise en œuvre et produira des discriminations subtiles (relatives aux friandises, aux jeux, aux corvées, aux réprimandes, aux récompenses, etc.).

L'anthropologue n'est pas le seul qui mette (parfois) en évidence ces normes pratiques discrètes et silencieuses. Bien souvent, la «sagesse populaire " (qui est en fait une philosophie et une sociologie populaire) évoque certaines d'entre elles, en tout cas les plus récurrentes, ne serait-ce qu'à travers la sémiologie quotidienne, comme nous l'avons vu ci-dessus. Le contraste baab-izey/nya-izey omniprésent dans le langage quotidien est en effet en opposition frontale avec la norme familiale/religieuse de l'harmonie de la famille polygame et du traitement indifférencié des frères (sœurs) et des demi-frères (sœurs). On sait que cette sagesse populaire s'exprime en particulier à travers fables et dictons, qui sont souvent plus enclins à décrire des pratiques effectives qu'à légitimer des normes morales (c'est un de leurs mérites). On ne s'étonnera donc pas qu'un proverbe songhay-zarma (yaasey) soit au rendez-vous concernant notre thème : «La fille de ta co-épouse ne va jamais te faire de belles tresses $»^{14}$. D'autres proverbes, nombreux, se réfèrent plus généralement à l'omniprésence de conflits au sein de la famille : «C'est l'enfant que tu portes dans ton dos qui peut te mordre»; «C'est avec la vache de ton père que tu apprends à faire le boucher »; «Le mariage ne peut pas être un bonheur plusieurs jours de suite $»^{15}$.

Certes, les rapports entre les co-épouses et entre leurs enfants sont, dans l'espace domestique, les plus susceptibles d'être conflictuels. Mais d'autres rapports intra-familiaux peuvent aussi être lourds de frustrations, de brimades, de violences ou de déviances, malgré leur régulation par des normes sociales de respect, de préséance ou de soumission (fort explicites et fréquemment invoquées). Il en est ainsi des rapports belle-mère/belles-filles (qui sont aussi des rapports de coexistence parfois difficiles dans une même unité de résidence),

13. Sur les normes pratiques en général (et plus particulièrement au sein de l'action publique), voir DE HERDT \& OLIVIER DE SARDAN (2015). Un exemple déjà ancien de norme pratique régulant les écarts à une norme sociale morale est fourni par D. KINTZ (1987), à propos de «l'art» de commettre l'adultère chez les Peuls du Nord-Burkina Faso.

14. "Weyce-ize si boro tuuru a ma bori."

15. " Izo kan ni hampa, nga dey no gin nama » ; «fawa wo, fu dey no ni ga dondon »; " hiijey si foy ga kaanu ». 
des rapports aîné/cadets, et des rapports mari/femme $(\mathrm{s})^{16}$. Tous sont porteurs d'écarts plus ou moins cachés aux normes sociales de référence.

Bien évidemment, il ne faut pas tordre le bâton à l'excès dans l'autre sens, et ne voir dans les familles que concurrences, intrigues, coups bas, vengeances, médisances ou trahisons. Les relations de fraternité, de soutien, de protection, de solidarité, ou d'entraide y sont aussi présentes. Haines et affections coexistent en permanence. Ce sont ces deux aspects qu'il faut considérer, et c'est cette ambivalence fondamentale qu'il faut prendre en compte, sans la réduire à l'un ou l'autre de ses pôles. Mais c'est loin d'être le cas, et le bâton est toujours dans un seul sens : la famille africaine reste très généralement perçue de façon idéalisée comme étant le site typique des nya-izey, en laissant dans l'ombre les baab-izey ${ }^{17}$.

\section{Extension aux relations de proximité}

L'opposition baab-izey/nya-izey est loin de rester cantonnée à l'univers familial (ou à celui de la famille chefferiale qui se situe déjà dans l'espace public). Elle est en effet utilisée de façon métaphorique dans le langage courant. Ceci n'a rien d'étonnant: le vocabulaire de la parenté est largement recyclé dans les relations interpersonnelles. Par exemple « tantie » ou « tonton » sont devenus des marques de respect envers un(e) ainé(e), utilisées couramment dans les rapports de travail comme dans les rapports de voisinage; de même, des expressions comme « grand frère », « ma sœur », « mon fils » sont couramment adressées à des proches ou à des moins proches sans aucun lien familial ${ }^{18}$. Quant à la fameuse parenté à plaisanterie, qui fonctionne au départ sur la matrice des relations entre cousins croisés, elle est invoquée quotidiennement, y compris entre inconnus, en référence à quelques vastes appartenances « ethniques » qui traversent la société nigérienne ${ }^{19}$.

16. Sur la dureté des rapports « amoureux » au Mali, voir par exemple DuMESTRE \& TouRÉ (1998).

17. Soyons clair : cette ambivalence des rapports intra-familiaux existe tout autant en Europe qu'en Afrique. Mais l'idéalisation de la famille a depuis longtemps été battue en brèche en Europe (même si elle rebondit régulièrement), et elle y fait l'objet de critiques souvent violentes (voir la phrase célèbre d'A. Gide « famille, je vous hais », ou le roman d'H. Bazin Vipère au poing). Par ailleurs, les réseaux familiaux et les contextes sociaux et culturels sont bien évidemment différents.

18. On peut aussi évoquer l'usage banalisé de «papa» en RDC qui correspond presque à «monsieur» en Europe.

19. Comme Songhay/Touaregs ; Zarma/Mawri ; etc... Ceci rappelle bien sûr (bien que de façon moins structurée) la senankuya mandingue. 
Cette parenté à plaisanterie entre groupes sociaux est justement souvent analysée comme un procédé cathartique permettant de désamorcer les tensions sociales. L'usage quasi ritualisé avec des inconnus ou des relations lointaines d'un franc-parler décontracté d'origine familiale pacifierait les relations interpersonnelles, d'interaction ou de circulation (Goffman 1983). Tout se passerait comme si la «parentalisation» de rapports de proximité avait un effet apaisant incorporé. On n'est pas loin de retrouver ici une idéologie de l'idéalisation de la famille, qui en exporterait les bénéfices, par son extension sémantique, à des relations extra-familiales. Mais s'il est vrai que la parenté à plaisanterie peut rendre amusantes les relations quotidiennes, adoucir certaines aspérités, ou masquer certains conflits, on ne doit jamais oublier qu'il s'agit là d'un mécanisme social d'étouffement (provisoire) des tensions (quand elles restent bénignes), et nullement d'un mécanisme de résolution des conflits. Les relations à plaisanteries relèvent d'un « mode mineur» de la réalité (Piette 1992), qui a son importance, mais qui ne doit pas être surestimé.

En fait, l'exportation à l'environnement social non familial de l'opposition baab-izey/nya-izey est là pour nous rappeler que les relations de proximité sont tout aussi ambivalentes que les relations familiales. Où que ce soit, dans toutes les relations interpersonnelles pourtant dénuées de liens de parenté (école, travail, sport, commerce, voisinage, associations...), baab-izey connote la jalousie et la rivalité, et nya-izey connote l'entraide et la solidarité. Autrement dit, les conflits de proximité sont systématiquement exprimés à travers la référence aux conflits entre demi-frères et demi-sœurs. Comme la famille, la socialité de proximité est tout autant un espace d'affrontements qu'un espace d'alliance. Les unités sociales d'interconnaissance ou les institutions et organisations locales ne sont pas seulement des ensembles soudés par une cause commune, présentant une façade de cohésion, et s'opposant à d'autres ensembles analogues (contradictions externes), ce sont aussi des ensembles traversés simultanément de tensions intestines (contradictions internes) ${ }^{20}$. Comme cela a déjà été souligné, les "face to face societies » sont aussi des «back to back societies » (Bailey 1965 : 5-7, cité dans Le Meur 2015: 413).

On ne s'étonnera donc pas de ce que les proverbes, là aussi, s'attachent à décrire les rivalités entre personnes proches : «Si les pieds sont près l'un

20. Certaines idéologies idéalisent les relations de proximité (comme d'autres, ou les mêmes, idéalisent la famille) : voir small is beautiful, le don local face au marché global, le voisinage ou l'associationnisme comme valeurs en soi, etc... 
de l'autre, ils se font des croche-pieds »; "Si tu te fâches avec tes fesses, tu devras t'assoir sur ton coccyx $»^{21}$.

Les conflits de proximité dans le monde politique

Nous voilà revenu à notre point de départ : le microcosme politique au Niger. À propos des politiciens, on parle fréquemment de baab-izey et, il faut le reconnaitre, fort peu de nya-izey. La rivalité et la jalousie sont des composantes structurales du monde politique ${ }^{22}$. Mais attention: il ne s'agit pas là de désigner ainsi la compétition entre partis politiques (elle n'a rien que de très normal, sous toutes les latitudes), mais les rivalités au sein des partis, qui sont un trait structurant de la politique nigérienne ${ }^{23}$. Les biographies de militants et de dirigeants sont ainsi révélatrices d'un «nomadisme » politique exacerbé : très nombreux sont ceux qui ont transité par trois ou quatre partis, parfois plus. Le terme de «transhumance» est régulièrement employé au Niger pour désigner ce phénomène ${ }^{24}$. Tout dirigeant, que ce soit à l'échelle locale ou nationale, s'il est mécontent des décisions de son parti, n'hésitera pas à le quitter avec toute sa clientèle électorale pour un autre parti, parfois ennemi déclaré du précédent, ou pour en fonder un nouveau ${ }^{25}$. Les raisons de ces scissions et changements de veste quasi permanents sont presque toujours de type personnel, et ne relèvent que très rarement de divergences programmatiques ou de désaccords idéologiques. N'avoir pas été désigné tête de liste pour des élections, n'avoir pas obtenu d'être maire, n'avoir pas été affecté à la tête de tel service ou de telle direction, n'avoir pas été affecté à

21. «Za ce hinka maanu cerga no i ga kati cerga »; « Ndin futu ni tundey se, kala ni ma goro ni banda biro gaa ».

22. Pour des exemples de conflits politiques locaux au Niger correspondant au modèle des baab-izey, voir MoHAMAdOU (2005) pour la région d'Abalak, BAKO ARIFARI (2013) pour la région de Gaya.

23. Bien sûr, les luttes de factions ou les guerres des chefs au sein d'un parti se retrouvent partout (comme en France). En ce domaine comme en d'autres, il n'y a pas de différence de nature entre ce qui se passe au Niger (et qui est assez semblable dans la plupart des pays africains) et ce qui se passe en Europe (ou aux États-Unis). Mais les proportions et le style varient en revanche beaucoup selon les contextes historiques. Par exemple, le «nomadisme politique» est bien plus développé au Niger qu'en France, et le Niger ne connait guère les combats idéologiques de l'hexagone.

24. Voir Gazibo (2005) et Olivier de SARdan (2015b). Ce phénomène a été décrit dans divers pays africains, voir par exemple GAZIBO (2003), LINDBERG (2003) et BIERSCHENK (2006).

25. On trouvera des exemples significatifs d'affrontements au sein d'un même parti politique à l'occasion de la constitution de listes pour des élections locales au Niger dans TIDJANI Alou (2015), Makama Bawa (2015) et Mohamadou \& Moha (2015). 
un poste rémunérateur, n'avoir pas été nommé ministre à la tête d'un ministère « juteux $»^{26}$, n'avoir pas obtenu un marché public qui récompensait vos services : voilà autant de motifs déclarés de ruptures, de virages ou de changements de parti dont l'actualité politique au Niger est remplie et où chacun peut reconnaître des cas récents. Nous avons relevé par exemple, entre 2004 et 2009, que dans près des deux-tiers des communes il y a eu des tentatives de destitution du maire, émanant le plus souvent de l'intérieur de sa majorité politique (Olivier de Sardan 2015b : 29) ${ }^{27}$.

Les conflits politiques au Niger sont donc avant tout des conflits personnels (où l'on implique ses amis et ses soutiens), comme ce sont des conflits de proximité (au sein d'un même parti, voire parfois d'une même faction). Ce sont des conflits de baab-izey. Tout camarade de parti est ainsi susceptible de devenir demain un ennemi politique, tout camarade de lutte peut se métamorphoser en rival acharné. Toute alliance est réversible à tout moment, ou presque. Le proverbe a plus raison encore pour la politique que pour la vie courante : «Partout où tu as un ami, tu as aussi un ennemi $»^{28}$. L'un peut à tout moment devenir l'autre.

On doit rappeler ici que les conflits de chefferie établissent en quelque sorte une liaison directe entre le monde de la famille et le monde de la politique. L'élection d'un chef de canton, marquée par les rivalités entre « vrais » baab-izey concurrents, est un événement politique national au Niger. Et, plus généralement, c'est toute la culture politique nigérienne qui est marquée par le modèle chefferial. Les chefs de parti, les ministres, les directeurs, les chefs de service, tendent tous plus ou moins à se comporter comme des chefs de canton, comme si l'entité à la tête de laquelle ils sont était leur propriété privée, avec toutes les caractéristiques de clientélisme, de courtisanerie et de confusion entre bien public et bien privé qui sont des traits structurels du mode chefferial de gouvernance (Olivier de Sardan 2009). Il faut ajouter que, du côté des populations, la demande correspondant à cette offre existe aussi, et qu'on attend des faveurs du chef de service comme du ministre, pour peu qu'on puisse établir un quelconque lien avec l'un ou l'autre, aussi indirect soit-il. Bien sûr, parmi toutes les caractéristiques du modèle chefferial étendu à la gouvernance étatique, figurent aussi les rivalités de type baab-izey.

Il s'agit donc plus que d'un simple emprunt sémantique au vocabulaire de la famille. Le vocabulaire du baab-ize tarey n'est pas exporté vers un univers différent sur un mode purement métaphorique. Il s'agit plutôt d'une

26. Cette expression locale signifie « qui offre des opportunités d'enrichissement ».

27. Pour des exemples précis, voir Abdoulkader (2008), Hahonou (2015), Moussa IBRAhIM (2015), IsSALEy \& OLIVIER DE SARDAN (2015).

28. "Nankan ni bako go kul no ni wanga go ». 
affinité structurelle entre famille et politique. Dans ces deux univers règnent des processus de même ordre. On en trouve une confirmation avec un autre terme « familial » qui est utilisé lui aussi dans la sphère politique : on appelle parfois « co-épouse » (weyce) un camarade-rival, un allié-rival (peu importe le genre). Les hommes politiques reproduisent entre eux les conflits de co-épouses (weyce) comme les conflits d'enfants de co-épouses (baab-izey). Ces antagonismes plus ou moins masqués sont à divers égards identiques dans l'univers domestique et dans l'univers des partis. Les conflits de proximité au sein de l'élite politique relèvent du même registre que les conflits entre co-épouses. On peut aussi employer le concept d'arène, inspiré de Bailey (1969), que nous avons souvent utilisé dans le cadre de la socio-anthropologie du développement (Bierschenk \& Olivier de Sardan 2003) : la famille étendue, comme l'élite politique, constituent deux arènes relativement similaires, où s'entrelacent, autour d'enjeux de pouvoir et de captation, antagonismes et solidarités entre acteurs proches relevant d'une même appartenance.

Les pratiques népotiques, si fréquentes dans le monde politique africain (mais qui existent bien sûr ailleurs) sont une illustration de cette homologie. Elles sont censées introduire de la solidarité familiale dans le monde cruel de la politique, et créer ainsi des enclaves de confiance. Mais elles y introduisent en même temps tensions familiales et querelles de fratries, tout en avivant les jalousies politiques. Loin d'apaiser les rivalités de proximité, le népotisme les exacerbe.

\section{Et les entrepreneurs magico-religieux?}

Au-delà de ces proximités sémiologiques et de ces affinités structurelles entre le monde de la famille, le monde des relations d'interconnaissance et le monde de la politique, tous marqués par la coexistence instable de l'amitié et de l'inimitié, de l'entraide et de la rivalité, de l'alliance et du déchirement, on peut aussi distinguer un autre facteur commun, peut-être plus inattendu, mais qui joue un rôle actif dans l'exacerbation des antagonismes de proximité. Il s'agit des « entrepreneurs magico-religieux» (des spécialistes de l'occulte). Nous utilisons ici un terme technique et moralement neutre, mais ils sont désignés au Niger sous divers noms : en français, féticheurs, devins, charlatans ou marabouts; en songhay-zarma, zimma (prêtres des génies et des danses de possession), alfaga (lettré coranique, ouléma), gunakoy (devin)... Tous ces spécialistes de l'occulte, jouant parfois la carte de l' «animisme » (en y incluant la géomancie comme diverses formes de polythéismes préislamiques), parfois la carte d'un certain islam, parfois les deux, sont en effet des recours 
permanents face aux complots supposés des proches, et alimentent à leur tour les soupçons contre l'entourage.

Là encore, la famille polygame est au centre. Les épouses sont une clientèle assidue de ces magiciens, à qui elles demandent non seulement d'avoir une grossesse réussie ou de garder l'amour de leur mari, mais aussi de les protéger contre les manœuvres supposées de leurs co-épouses. D’ailleurs, parfois, on sollicitera soi-même qu'une rivale avorte ou soit répudiée par le mari. Très nombreuses sont les femmes qui vont voir en catimini un entrepreneur magico-religieux pour qu'il rende leur mari « doux comme un mouton » et qu'il obéisse désormais à tous leurs désirs. Ces commerçants en pratiques occultes ont bien sûr toujours en stock les « fétiches », les charmes magiques et les talismans nécessaires, ils ne manquent pas d'incantations, de rituels, de versets ou de sacrifices, bénéfiques ou maléfiques, pour se défendre comme pour attaquer. Mieux, ce sont eux qui induisent la mécanique du soupçon, de la défense et de la contre-attaque. Une femme vient les voir : elle est malade de façon inexpliquée, ou ses enfants ne travaillent pas bien à l'école, ou elle a fait une fausse-couche, ou son commerce périclite. Tout devin, tout magicien qui se respecte aura toujours le même type d'explication : c'est ta co-épouse qui t'a « maraboutée » (ou qui t'a envoutée, ou qui a fait « travailler » contre toi un autre magicien, etc.) ; c'est ta « rivale » qui t'a « envoyé » tous ces malheurs...

Passons maintenant au monde politique. Une élection se profile : les hommes politiques se pressent à leur tour discrètement chez les magiciens. Pour gagner, éliminer leurs concurrents. Mais aussi pour savoir s'il n'y a pas de traître dans leurs rangs. Pour que leurs soutiens ne les abandonnent pas, et que leurs amis ne retournent pas leur veste. Ici aussi, les magiciens induisent la mécanique du soupçon : attention, dans ton entourage il y a quelqu'un qui veut ta place.

Dans l'espace familial, comme dans l'espace politique, il est presque impossible d'échapper à ces consultations discrètes et à ces pratiques occultes. Il ne s'agit même pas tant d'y croire ou pas ${ }^{29}$. L'entourage y pousse. Ce sont les sœurs ou les amies qui amènent l'épouse chez le zimma. Refuser serait signe de mépris ou de stupidité. Ce sont les supporters et les conseillers qui organisent la visite de l'homme politique chez le marabout ou le devin. Tout candidat aux élections se doit de subventionner des étudiants coraniques pour lire intégralement et publiquement le Coran en sa faveur, mais aussi d'aller nuitamment voir un expert en pratiques magiques ${ }^{30}$. Ne pas agir dans ce

29. Il s'agit de ce qu'on a appelé une «attitude naturelle» (SCHUTZ 1971). Voir également Olivier De SARDAN (1992).

30. Voir le cas d'un candidat à la députation dans MAKAMA BAWA (2015). 
registre serait désavouer ses partisans, renier les coutumes locales, se couper de ses électeurs, et vouer son entreprise politique à l'échec.

Dans les deux cas, c'est un cycle infernal. Les rivalités familiales et les rivalités politiques de proximité enclenchent un recours aux entrepreneurs magico-religieux, qui renforcent les soupçons pesant sur l'entourage, qui accentuent les rivalités. Le baab-ize tarey prospère.

On pourrait d'ailleurs appliquer le modèle du baab-ize tarey aux entrepreneurs magico-religieux eux-mêmes : la compétition est en effet sévère entre eux, ce qui explique d'un côté la fragmentation du marché des « féticheurs » et « guérisseurs », comme d'un autre côté la fragmentation de la sphère islamique. Il s'agit, dans les deux cas, de conflits de proximité.

Cette exacerbation des accusations envers l'entourage dans un registre de l'occulte a déjà bien évidemment été analysée, soit du côté de la psychiatrie/ psychanalyse, soit du côté de l'anthropologie des rapports entre sorcellerie et politique. Je n'ai pas la place ici de discuter en profondeur ces interprétations, et je me contenterai de donner deux pistes

Une certaine disposition « paranoïaque » spécifique a souvent été relevée lors d'études sur les troubles mentaux en Afrique (Ortigues \& Ortigues 1966; Zempléni 1975). Il s'agit presque toujours de mises en cause de l'entourage, soupçonné d'attaques mystiques ou sorcellaires. Plutôt que de se référer à une contestable "personnalité de base » africaine ou à une quelconque culture traditionnelle, il faut souligner le rôle important que jouent (au moins au Niger) ces deux institutions contemporaines que sont, d'une part, la famille polygame ${ }^{31}$ et, d'autre part, les entrepreneurs magico-religieux qui alimentent, bien en amont des cas pathologiques décrits par les psychiatres, une fabrique permanente du soupçon de proximité, au cœur des relations quotidiennes dans les espaces familiaux et les espaces de pouvoir.

D'un autre côté, certains anthropologues ou politologues se sont focalisés sur le rôle de la sorcellerie (plus exactement des accusations de sorcellerie) et donc de la «dévoration » dans la vie politique, en particulier dans un contexte camerounais, comme expression de la quête de pouvoir (ou parfois, et inversement, comme anti-pouvoir), et ils en ont fait à divers égards une

31. La polygamie remonte, certes, à la période pré-coloniale. Mais, au sein de la classe politique, c'est une construction post-coloniale récente. À l'indépendance et dans les deux décennies qui ont suivi, la très grande majorité des élites nigériennes était monogame. Aujourd'hui, c'est l'inverse : la très grande majorité est polygame. La monté en puissance $\mathrm{du}$ fondamentalisme islamique n'est sans doute pas étrangère à cette évolution. 
dimension centrale de la culture politique africaine : «manger l'âme d'autrui » est perçu comme une condition de l'accès au pouvoir, qui permet à son tour le pillage des fonds publics, lui-même caractérisé comme le fait de «manger » (Bayart 1989; Geschiere 1997). Mais, outre le fait que le contexte camerounais est à cet égard très différent du contexte nigérien (et ne peut être généralisé au continent), il nous semble difficile de lire la politique « à travers » les pratiques occultes, comme si ces dernières avaient un rôle explicatif ${ }^{32}$. Nous verrions plutôt dans les pratiques occultes un «accélérateur» des caractéristiques agonistiques de la vie politique.

Le baab-ize tarey, c'est-à-dire la relation de rivalités entre frères, est une réfutation empirique des nombreux écrits sur la politique en Afrique, qui caractérisent cette dernière comme dominée par les solidarités traditionnelles, les allégeances familiales ou ethniques, sans place pour l'individu (Chabal \& Daloz 1998 ; Schatzberg 2001) ${ }^{33}$. La réalité est toute différente : les conflits individuels et les stratégies personnelles sont en première ligne, selon l'avis d'une majorité de citoyens. Bien sûr, les relations de clientèle, les réseaux, les allégeances collectives et les solidarités de proximité existent aussi. Mais les deux dimensions doivent être prises en considération.

C'est parce que la politique au Niger se réduit pour l'essentiel à des conflits incessants de personnes et de factions sans perspectives plus vastes qu'elle peut ainsi se résumer à des intrigues de baab-izey, avec tout ce que cela implique, y compris le recours aux entrepreneurs magico-religieux. Mais c'est justement le reproche qui est fait à l'élite politique par une partie de plus en plus importante de la population : cette élite ne pense qu'à elle-même, n'est intéressée que par le gain du pouvoir ou son maintien au pouvoir, et ne songe qu'à s'enrichir. Ce constat alimente une opinion largement répandue : la démocratie introduite dans la fin des années 1980 est un échec.

À cet échec, certains opposent le sens perdu du bien public, le besoin de renouveler les élites, l'urgence de développer l'esprit civique, l'impérieuse nécessité d'une réforme de l'État. La figure de Thomas Sankara, devenue mythique en Afrique de l'Ouest, est souvent mobilisée dans cette perspective. D'autres, au contraire, rêvent de charia, de gouvernance islamique, d'islam politique, voire de jihad. C'est une autre figure mythique, celle du caliphe, qui est alors mobilisée.

32. Pour une critique de cette « construction de l'occulte », voir RANGER (2007); pour une critique de l'argumentation sur la « dévoration», voir BLUNDO \& OLIVIER DE SARDAN (2006).

33. Pour une critique de ce « culturalisme traditionnaliste africaniste », voir OLIVIER DE SARDAN (2010). 
Ce sont là deux façons opposées de rompre avec le baab-ize tarey généralisé du tout petit monde politique nigérien actuel. On peut aussi penser que cette analyse vaut pour les pays voisins du Sahel, qui non seulement ont un modèle émique analogue des relations intra-familiales, mais qui connaissent aussi un même type de rivalités politiques, et la même crise de la démocratie.

Laboratoire d'études et de recherches sur les dynamiques sociales et le développement local (LASDEL), Niamey, Niger.

\section{BIBLIOGRAPHIE}

Abdoulkader A., 2008, « La commune d'In Gall », Études et Travaux du LASDEL, 73.

ANDERS G., 2010, In the Shadow of Good Governance. An Ethnography of Civil Reform in Africa, Leiden, Brill.

Bailey F., 1965, «Decisions by Consensus in Councils and Committees. With Special Reference to Village and Local Government in India », in M. BANTON (ed.), Political Systems and the Distribution of Power, London, Tavistock.

— 1969, Stratagems and Spoils, London, Basil Blackwell.

BAKO ARIFARI N., 2013, Le pouvoir politique en milieu rural africain, Cotonou, Éditions Afridic.

BAyART J.-F., 1989, L'État en Afrique. La politique du ventre, Paris, Fayard.

Bierschenk T., 2006, «The Local Appropriation of Democracy: An Analysis of the Municipal Elections in Parakou, Republic of Benin, 2002-03», Journal of Modern African Studies, 44 (4) : 543-571.

Bierschenk T. \& Olivier de SARdan J.-P., 2003, « Powers in the Village. Rural Benin between Democratisation and Decentralisation », Africa, 73 (2) : 145-173.

Blundo G. \& Olivier de SARdan J.-P., 2006, « The Popular Semiology of Corruption », in G. Blundo \& J.-P. Olivier de SARdan (eds.), with N. Bako Arifari \& M. Tidjani Alou, Everyday Corruption and the State. Citizens and Public Officials in Africa, London, Zed Books.

CANUt C. \& SMith S. (DIR.), 2006, numéro spécial « Parentés, plaisanteries et politique », Cahiers d'Études africaines, XLVI (4), 184. 
Chabal P. \& Daloz J.-P., 1998, Africa Works : Disorder as Political Instrument, Oxford, James Currey.

Courade G., 2006, L'Afrique des idées reçues, Paris, Belin.

De Herdt T. \& Olivier de Sardan J.-P. (eds.), 2015, Real Governance and Practical Norms in Sub-Saharan Africa. The Game of the Rules, London, Routledge.

Dumestre G. \& Touré S., 1998, Chroniques amoureuses au Mali, Paris, Karthala.

FAINZANG S. \& Journet O., 1988, La femme de mon mari. Anthropologie du mariage polygamique en Afrique et en France, Paris, L'Harmattan.

GAZIBO M., 2003, «Les vertus des procédures démocratiques. Élections et mutations du comportement politiques au Niger », Politique Africaine, 92 : 145-156.

—2005, Les paradoxes de la démocratie en Afrique. Analyse institutionnelle et stratégique, Montréal, Presses universitaires de Montréal.

Geschiere P., 1997, The Modernity of Witchcraft. Politics and the Occult in Post-colonial Africa, Charlottesville, University Press of Virginia.

Goffman E., 1983, « The Interaction Order », American Sociological Review, 48 (1) : 1-17.

Hahonou E., 2015, «Les élections municipales de 2009 à Téra : les frères ennemis », in J.-P. OlIVIER DE SARDAN (dir.), Élections au village. Une ethnographie de la culture électorale au Niger, Paris, Karthala : 189-207.

IsSAley N. \& Olivier De SARDAN J.-P., 2015, « Les élections locales à Namaro en 2004 », in J.-P. OLIVIER DE SARDAN (dir.), Élections au village. Une ethnographie de la culture électorale au Niger, Paris, Karthala : 255-272.

KINTZ D., 1987, « De l'art peul de l'adultère », Bulletin de l'AFA, 29-30 : 119-143.

Le Meur P.-Y., 2015, « Anthropology and the Mining Arena in New Caledonia : Issues and Positionalities », Anthropological Forum, 25 (4) : 405-427.

Lindberg S., 2003, " "It is Our Time to Chop" : Do Elections in Africa Feed Neopatrimonialism Rather than Counteract It ? », Democratization, 10 (2) : 121-140.

Makama Bawa O., 2015, «"Saï Kaayi!” ou comment se faire élire au Niger. Analyse des stratégies électorales d'un candidat aux législatives 2009 », in J.-P. OLIVIER DE SARDAN (dir.), Élections au village. Une ethnographie de la culture électorale au Niger, Paris, Karthala : 75-106.

Mohamadou A., 2005, «Les pouvoirs locaux dans la commune d'Abalak », Études et Travaux du LASDEL, 34.

Mohamadou A. \& Moha M., 2015, «Les élections locales dans le canton de Birnin Lallé », in J.-P. OLIVIER DE SARDAN (dir.), Élections au village. Une ethnographie de la culture électorale au Niger, Paris, Karthala : 137-172. 
Moussa Ibrahim H., 2015, «Les élections municipales en 2009 et 2011 ; stratégies électorales et recomposition des arènes locales », in J.-P. Olivier DE SARDAN (dir.), Élections au village. Une ethnographie de la culture électorale au Niger, Paris, Karthala : 313-329.

Olivier de SARdan J.-P., 1982, Concepts et conceptions songhay-zarma (histoire, culture, société), Paris, Nubia.

— 1984, Les sociétés songhay-zarma. Chefs, esclaves, guerriers, paysans..., Paris, Karthala.

— 1992, «Occultism and the Ethnographic "I". The Exoticizing of Magic from Durkheim to Post-modern Anthropology », Critique of Anthropology, 12 (1) : 5-25.

- 2009, «Gouvernance associative et gouvernance chefferiale dans les campagnes nigériennes », in J.-P. Olivier DE SARDAN \& M. Tidjani Alou (dir.), Les pouvoirs locaux au Niger. En attendant la décentralisation, Paris, Karthala.

- 2010, «Le culturalisme traditionnaliste africaniste. Analyse d'une idéologie scientifique », Cahiers d'Études africaines, L (2-3-4), 198-199-200 : 419-453.

- 2015a, «Practical Norms : Informal Regulations within Public Bureaucracies (in Africa and beyond) », in T. De HerdT \& J.-P. Olivier DE SARDAN (eds.), Real Governance and Practical Norms in Sub-Saharan Africa. The Game of the Rules, London, Routledge : 19-62.

— 2015b, «Une culture électorale ? Anthropologie multi-sites des processus électoraux locaux au Niger », in J.-P. Ollvier DE SARDAN (dir.), Élections au village. Une ethnographie de la culture électorale au Niger, Paris, Karthala : 9-49.

— 2016, «Les quatre prisons du pouvoir», Marianne, 4 janvier 2016, <https://www. marianne.net/debattons/tribunes/niger-les-quatre-prisons-du-pouvoir > (publié par Le Républicain, Niamey, 31 décembre 2015).

— 2017, «Rivalries of Proximity beyond the Household in Niger : Political Elites and the baab-izey Pattern » Africa, 87 (1) : 120-136.

Olivier de Sardan J.-P. \& Tidjani Alou M. (DIR.), 2009, Les pouvoirs locaux au Niger. En attendant la décentralisation, Paris, Karthala.

Ortigues M. C. \& Ortigues E., 1966, Oedipe africain, Paris, Plon.

Piette A., 1992, Le mode mineur de la réalité. Paradoxes et photographies en anthropologie, Louvain, Peeters.

Ranger T. O., 2007, « Scotland Yard in the Bush : Medicine Murders, Child Witches and the Construction of the Occult : A Literature Review », Africa, 77 (2) : 272-283.

Schatzberg M. G., 2001, Political Legitimacy in Middle Africa : Father, Family, Food, Bloomington, Indiana University Press.

Schutz A., 1971, Collected Papers I: The Problem of Social Reality, The Hague, Martinus Nijhoff. 
Tidjani Alou M., 1992, Les politiques de formation en Afrique francophone. École, État et société au Niger, Thèse de doctorat, Bordeaux, Université de Bordeaux I.

TIDJANi Alou M., 2009, « La chefferie et ses transformations. De la chefferie coloniale à la chefferie post-coloniale », in J.-P. Olivier DE SARdAn \& M. Tidjani Alou (dir.), Les pouvoirs locaux au Niger. En attendant la décentralisation, Paris, Karthala : 37-62.

— 2015, «Une anthropologie de la politique au village», in J.-P. OLIVIER DE SARDAN (dir.), Élections au village. Une ethnographie de la culture électorale au Niger, Paris, Karthala : 51-73.

Zempleni A., 1975, « De la persécution à la culpabilité », in C. Piault (dir.), Prophétisme et thérapeutique. Albert Atcho et la communauté de Bregbo, Paris, Hermann : 153-178.

\section{RÉSUMÉ}

Aujourd'hui, au Niger, la politique des partis et de la démocratie, caractérisée par des affontements incessants entre politiciens, avec de fréquents changements de camp, est rejetée par la population. Ces conflits de proximité sont nommés par un terme emprunté aux conflits familiaux, qui s'applique aux relations de jalousie entre demi-frères, à savoir les baab-izey. La famille est un espace autant de rivalités que de solidarité, contrairement aux stéréotypes sur la famille africaine. Ces rivalités alimentées par la polygamie deviennent publiques dans les querelles d'héritage ou de chefferie. Le microcosme politique nigérien est confronté à des rivalités internes semblables où les camarades d'aujourd'hui deviennent demain des ennemis. Autre similarité, les conflits de proximité familiaux comme les conflits de proximité politiques sont avivés par les spécialistes de l'occulte qui rendent toujours les proches responsables des problèmes.

Mots-clés: Niger, chefferie, classe politique, conflits, demi-frères, démocratie, factions, famille, occulte, parenté à plaisanterie, polygamie.

\section{ABSTRACT}

Rivalries of Proximity and the Crisis of Democracy: From Household to Political Elites, in Niger and Beyond. - In Niger, there is an increasing rejection of party politics and the politics of democracy, characterized by personal rivalries. There is a direct link between the social perceptions of intra-familial rivalries and the social perceptions of political rivalries. The archetypical relationship among the baab-izey (children of one father but different mothers) is characterized by competition and jealousy, as a consequence of the latent rivalry that pits the co-wives against each other. The same is true for the political microcosm of Niger: political conflicts are above all personal/factional conflicts in which friends and supporters are implicated, and thus constitute rivalries of proximity. In the familial space as in the political space, experts in the occult are merely an "accelerator" of these conflicts: they reinforce suspicions about the familial or political entourage.

Keywords: Niger, chieftancy, conflicts, democracy, factions, joking relationship, occult, political elites, polygamy, siblings. 